\title{
ELT near-infrared and thermal-infrared studies of massive star formation: direct imaging and integral field spectroscopy of ultracompact HII regions
}

\author{
Hans Zinnecker \\ Astrophysikalisches Institut Potsdam, An der Sternwarte 16, 14482 Potsdam, Germany \\ email: hzinnecker@aip.de
}

\begin{abstract}
In this contribution, we show how a future ELT ( $>25 \mathrm{~m}$ diameter) helps to understand the formation and early dynamical evolution of massive stars embedded in dustenshrouded very compact HII regions. We describe how to exploit the ELT's near- and mid-IR enhanced sensitivity and high angular resolution to peer through huge amounts of dust extinction, taking direct nearly diffraction-limited images and doing IFU spectroscopy. Together with ALMA, an ELT will be a powerful observing platform to reveal one of the most hidden secrets of stellar astrophysics: the origin of massive stars.
\end{abstract}

Keywords. techniques: high angular resolution stars: formation stars: early-type ISM: dust, extinction ISM: HII regions infrared: stars

\section{Introduction}

Massive stars $\left(\mathrm{M}>20 \mathrm{M}_{\odot}\right)$ are beacons, wreckers, and cosmic engines of change within their galactic and extragalactic environment. When they die and explode as supernovae, they dramatically deposit lots of kinetic energy and chemically enriched material in the interstellar medium. Yet despite their enormous overall importance for galactic dynamical and chemical evolution, their origins and birth processes remain poorly understood (e.g. Bally \& Zinnecker 2005).

In this contribution, we try to show how future ELTs ( $>25$ m diameter) help to understand the formation and early dynamical evolution of massive stars embedded in highly obscured very compact HII regions. We suggest to exploit the ELT's near- and mid-IR enhanced sensitivity and angular resolution to peer through huge amounts of dust extinction, and take direct nearly diffraction limited infrared images, in order to find clear observational evidence for or against disk accretion and binary stellar collisions (two of the leading formation models).

\section{Science case for ultra compact HII regions}

Although a number of formation sites associated with ultracompact $(0.1 \mathrm{pc})$ and hypercompact $(0.01 \mathrm{pc})$ HII regions have been identified, the gas column density $\left(3 \cdot 10^{23} \mathrm{~cm}^{-2}\right)$ and dust extinction towards the exciting massive stars are too high $\left(\mathrm{A}_{V}>100\right)$ to detect their photospheres in the near-infrared $K$-band, even with current $10 \mathrm{~m}$-class telescopes. At the same time, the angular resolution is too low $\left(0^{\prime \prime} .1\right)$ for the typical distance (ca. $2-4 \mathrm{kpc}$ ) and the dense clustering of objects (several compact radio sources within $1-2^{\prime \prime}$ ) to spatially resolve the stellar substructure of these HII regions. An ELT can penetrate and resolve such massive star forming regions (with properties as in Faúndez et al. 2004). 
As an example (cf. W3-IRS5: Megeath et al. 2005, 1996, Claussen et al. 1994) we derive the scientific requirements for an ELT to resolve a cluster of four massive stars (Trapezium system) embedded in a dense hypercompact HII region with radius $1000 \mathrm{AU}$ at $2 \mathrm{kpc}$ distance (diameter $1^{\prime \prime}$ ) behind $200 \mathrm{mag}$ of visual extinction (22.5 mag extinction in the $K$-band at around $2 \mu \mathrm{m}$ and $4.5 \mathrm{mag}$ in the $M$-band at around $5 \mu \mathrm{m}$; based on the interstellar extinction law of Rieke \& Lebofsky 1985). Other extinction laws are discussed below.

\subsection{Requirements for infrared imaging (KLM)}

The direct imaging adaptive optics observations require a spatial resolution of 10 mas and need to reach a limiting apparent magnitude of $\mathrm{K}=29(\mathrm{M}=11)$, assuming an absolute magnitude of $M_{K}=-5$ of the massive stars in question and a distance modulus of 11.5. These requirements can be met by a giant telescope of $40-60 \mathrm{~m}$ diameter, if a natural near-IR bright guide star can be found, within $10-30^{\prime \prime}$, perhaps in the adjacent compact HII region, on which the wavefront sensing can be performed. Possibly, a laser guide star will be needed.

High proper motion (several mas/yr), including dynamical ejections at velocities in excess of $30 \mathrm{~km} \mathrm{~s}^{-1}$ due to close encounters (ca. $20 \mathrm{AU}$ ), in such multiple systems can be monitored by successive imaging over a $1-2$ year timeline.

\subsection{Requirements for infrared spectroscopy (IFU)}

Integral field spectroscopy of the complex interaction zone of the incipient cluster will also be most useful. The goal here would be to derive the extinction to the individual massive stars by measuring the flux ratio of the Brackett gamma $(2.17 \mu \mathrm{m})$ to Brackett alpha $(4.05 \mu \mathrm{m})$ line, which changes with extinction from its intrinsic Menzel Case B value $(1 / 3)$. Furthermore, we expect to detect collimated $\mathrm{H}_{2}$ jets $(2.12 \mu \mathrm{m})$ if the stars are in a phase of active disk accretion. Also, CO bandhead emission and absorption (both the fundamental at $4.6 \mu \mathrm{m}$ and the first overtone at $2.3 \mu \mathrm{m}$ ) must be searched for to determine the temperature of the warm gas near the stellar photosphere and its kinematics (rotation, infall). A velocity resolution of $30 \mathrm{~km} \mathrm{~s}^{-1}$ is required, i.e. $\mathrm{R}=10^{4}$. This would also allow us to detect radial velocity variations and orbital motion of massive close binaries, making use of near-IR photospheric absorption lines. Many visible massive OB stars are double-lined short-period spectroscopic binaries, but the question is whether they are born as close binaries and if so how (Bonnell \& Bate 2005).

The requirements on the integral field spectroscopy are as follows: we would hope to use $4 \mathrm{k} \times 4 \mathrm{k}$ IR-arrays, which at $5 \mathrm{mas} /$ pixel provide a $\mathrm{FoV}=20^{\prime \prime}$ for imaging, thus covering the wider cluster core $(0.1 \mathrm{pc})$. For IFU spectroscopy, we can concentrate the unit on the central $2^{\prime \prime} \times 2^{\prime \prime}$ field, requiring $400 \times 400=160000$ fibers. Given the desired spectral resolution of 10000 , we need 2000 pixels to disperse the whole $K$-band $(2.0-2.4 \mu \mathrm{m})$. If we use only $1 / 2$ of the $K$-band $\left(2.10-2.18 \mu \mathrm{m}\right.$ for $\mathrm{H}_{2}$ and $\mathrm{Br} \gamma$, and $2.29-2.31 \mu \mathrm{m}$ for 2-0 CO bandhand), we can fit 4 spectra in one spectral row (4000 pixels). As we have $4 \mathrm{k}$ spectral rows, we can fit $16 \mathrm{k}$ spectra or fibers on one IR array detector, hence we need $160000 / 16000=10$ IR chips to match our scientific requirements. Similarly for the $M$-band. Not impossible, by 2015 (but expensive: ca. $1 \mathrm{M} \mathrm{U} \$$ for a $4 \mathrm{k} \times 4 \mathrm{k}$ IR chip).

\subsection{Dependence on the infrared extinction law}

A crucial ingredient in all our considerations is the use of the "correct" extinction law, which depends on the properties and size distribution of the dust grains which in turn depend on the density of the star forming cloud. We used a ratio of optical-to-infrared extinction $\mathrm{A}_{K} / \mathrm{A}_{V}=0.112$ and $\mathrm{A}_{M} / \mathrm{A}_{V}=0.023$, respectively (Rieke \& Lebofsky 1985). 


\section{spectral dim (4 spectra per row)}

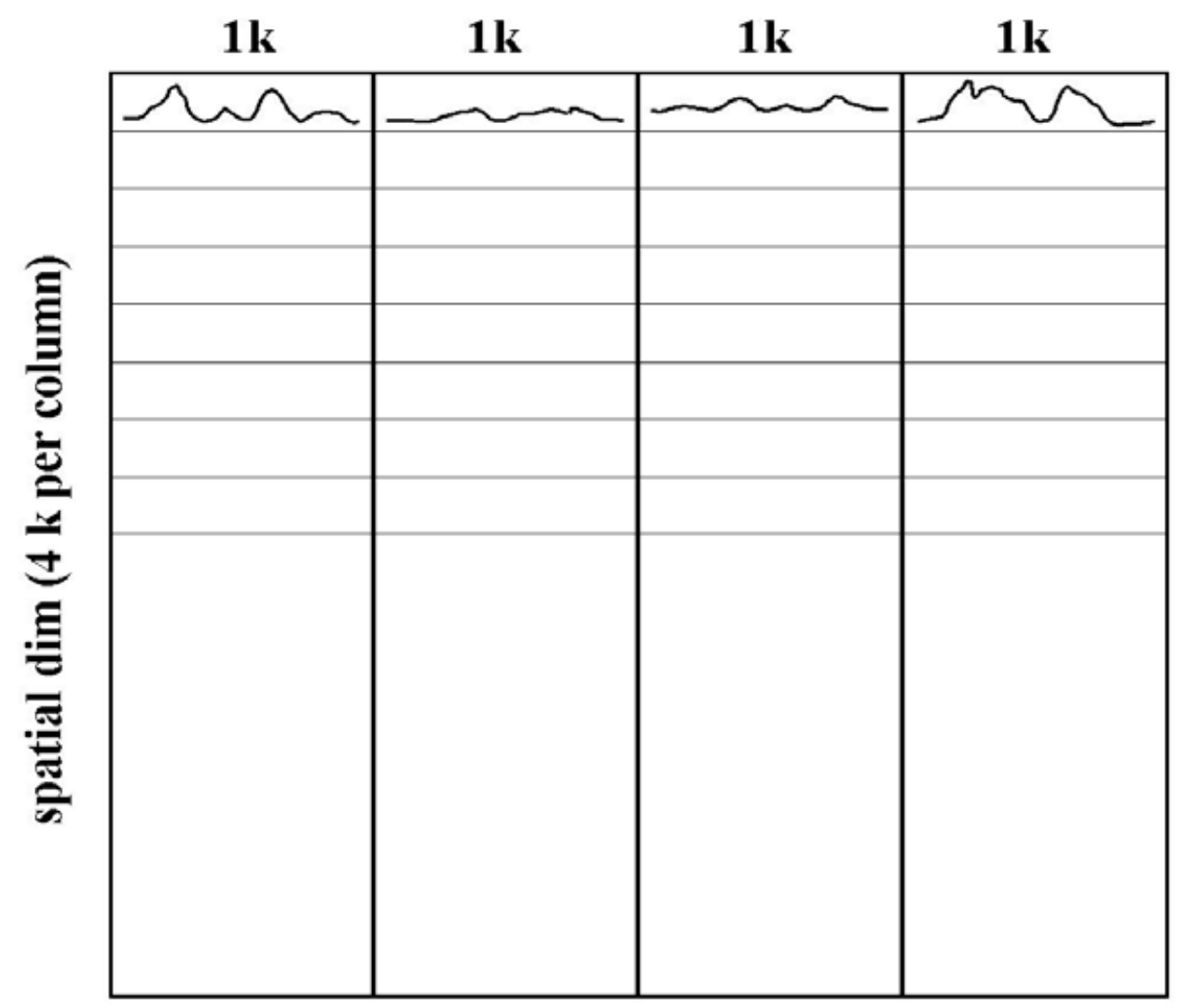

Figure 1. $4 \mathrm{k} \times 4 \mathrm{k}$ IR-array for IFU spectroscopy (see text). Spectral and spatial coordinates are indicated as rows and columns, respectively. A few artificial spectra (part of the $K$-band) are shown. Note that the present arrangement is different from the VLT/MUSE IFU concept.

However, different authors provide different relationships. While Landini et al. (1984) reconfirm the van de Hulst No. 15 relation (see Rieke and Lebofsky 1985) in their recombination line study of the famous southern HII region G333.6-0.2, Cardelli et al. (1989) argue for a flattened extinction curve in dense molecular clouds (with $\mathrm{A}_{K} / \mathrm{A}_{V}=0.114$ and $\mathrm{A}_{M} / \mathrm{A}_{V}=0.056$, see their Table 3). Possibly, there is a local minimum of the thermal IR extinction in the M-band (Lutz et al. 2002) which would help our ELT science case for thermal IR studies.

\section{Outlook (ELT \& ALMA)}

Finally we mention that ALMA will have a similar resolution of 10 mas at submm wavelengths as "our" ELT in the near- and thermal infrared. Hence, while ELT will be particularly good at detecting OB stars at the end of their main accretion phase, ALMA should see OB protostars, i.e. massive stellar objects in their early or main mass assembly phase. Together ELT and ALMA are a powerful combination to reveal one of the most hidden, but most important secrets of stellar astrophysics: the origin of massive stars. 


\section{Acknowledgements}

Travel support to this symposium was kindly provided by OPTICON. Helpful discussions with Tom Herbst (MPIA) and Martin Roth (AIP) are gratefully acknowledged, as are comments by Mark McCaughrean (the organisor of the parallel session on star formation and exoplanets in which this paper was presented). Last but not least, many thanks to the LOC for providing a free trip and a guided tour to the newly inaugurated Southern African Large Telescope (SALT).

\section{References}

Bally, J. \& Zinnecker, H. 2005, AJ 129, 2281

Bonnell, I.A. \& Bate, M.R. 2005, MNRAS 362, 915

Cardelli, J.A., Clayton, G.C. \& Mathis, J.S. 1989, ApJ 345, 245

Claussen, M.J., Gaume, R.A., Johnston, K.J. \& Wilson, T.L. 1994, ApJ (Letters) 424, L41

Faúndez, S., Bronfman, L., Garay, G., Chini, R., Nyman, L.-Å. \& May, J. 2004, A\& A 426, 97

Landini, M., Natta, A., Salinari, P., Oliva, E. \& Moorwood, A.F.M. 1984, A\& A 134, 284

Lutz, D., Maiolino, R., Moorwood, A.F.M., Netzer, H., Wagner, S.J., Sturm, E. \& Genzel, R. 2002, A $\mathscr{E} A$ 396, 439

Megeath, S.T., Herter, T., Beichman, C., Gautier, N., Hester, J.J., Rayner, J. \& Shupe, D. 1996, A $\mathscr{E} A$ 307, 775

Megeath, S.T., Wilson, T.L. \& Corbin, M.R. 2005, ApJ (Letters) 622, L141

Rieke, G.H. \& Lebofsky, M.J. 1985, ApJ 288, 618

\section{Discussion}

PUZIA: How exactly will ELT observations help to distinguish between the gas accretion and coalescence scenario of massive star formation?

ZINNECKER: An ELT will have very high angular resolution which will allow us to see if the formation sites of massive stars (i.e. UCHII) have the high stellar densities necessary for coalescence to occur.

BALEGA: Is the formation mechanism of the most massive stars different from that of other massive stars (e.g. in the Orion Nebula)?

ZiNNECKER: Perhaps yes. In a very dense protocluster the most massive star may form by coalescence of two massive stars, especially through the merging of a massive binary, located at the cluster center.

RouAn: Aren't you largely dominated in the thermal IR by hot dust emission around stars rather than by the stars themselves?

ZINNECKER: To some extent yes. However, the very high angular resolution adaptive optics observations will help to minimize this effect, favoring point sources and suppressing the extended emission.

ZIJLSTRA: Doesn't dust scattering affect (blur) the point spread function?

ZINNECKER: Good point. However, dust scattering is more important in the $J$ and $H$ bands than in the $K L M$-bands, and it is only the latter that we will/can use to penetrate the dense dusty cloud cores. 